\title{
Site Directed Disulfide PEGylation of Interferon-beta-1b with Fork Peptide
}

\section{Linker}

Shayan Abbasi, ${ }^{\dagger+}$ Homa Farahani, ${ }^{\S}$ Hossein Lanjanian, ${ }^{\dagger}$ Mohammad Taheri, ${ }^{\ddagger}$ Loghman

Firoozpour, ${ }^{\ddagger}$ Jamshid Davoodi, ${ }^{\dagger}$ Sama Pirkalkhoran, ${ }^{\ddagger}$ GholamHossein Riazi ${ }^{*, \dagger}$ and Shahriar

Pooyan*,†,+

$\dagger$ Institute of Biochemistry and Biophysics, PO Code 1417614335, University of Tehran, Tehran, Iran

Rooyan Darou Pharmaceutical Company, PO Code 15996-89111, Tehran, Iran

$\S$ Department of Microbiology, School of Biology, PO Code 1417466191, Faculty of Science, University of Tehran, Tehran, Iran

${ }^{¥}$ Department of Medicinal Chemistry, Faculty of Pharmacy and Pharmaceutical Sciences

Research Center, Tehran University of Medical Sciences, PO Code 14174, Tehran, Iran

${ }^{\ddagger}$ Department of Biology, Faculty of Basic Science, Islamic Azad University of Central Tehran Branch, PO Code 1477893855, Tehran, Iran

* Corresponding authors:

Shahriar Pooyan: Pooyan@rooyandarou.com

GholamHossein Riazi: Ghriazi@ut.ac.ir 
Figure S1. Determination of limit of detection and quantification

for fluorescamine and Aldrithiol assays.

Figure S2. Quantification of Core Peptide.

S3

Figure S3. Quantification of purified PCP.

Figure S4. Quantification of PCP-SPDP Conjugation (Crude).
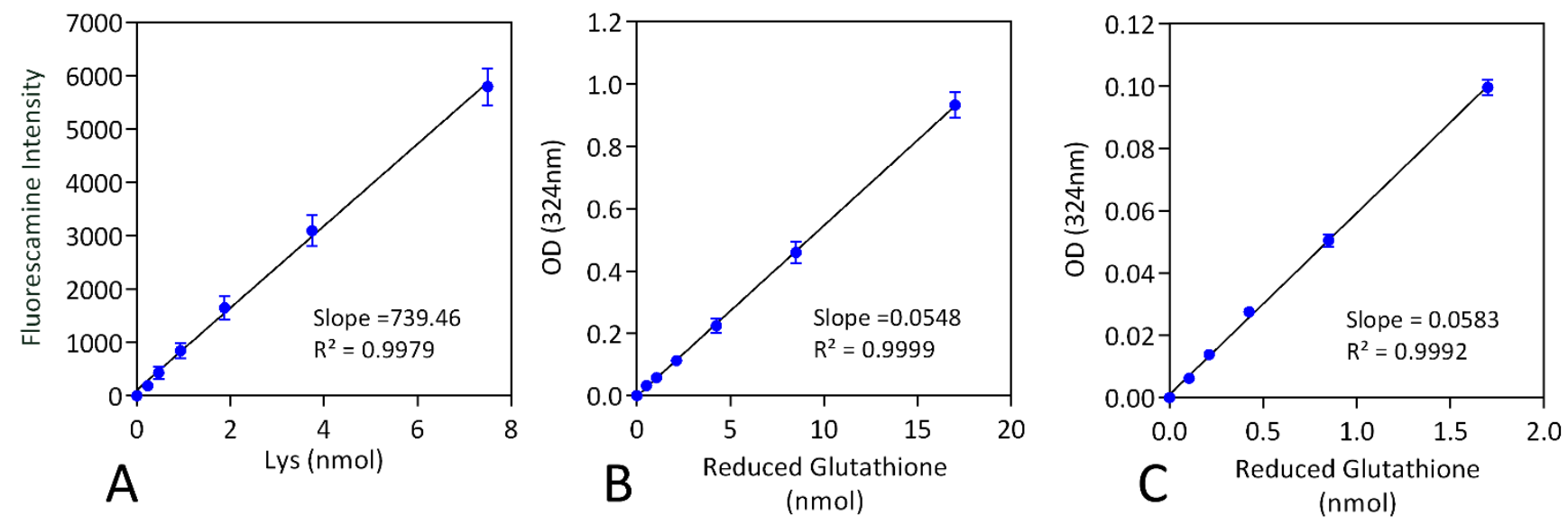

Figure S1. Determination of limit of detection and quantification for fluorescamine and Aldrithiol assays. The calibration curves were prepared as described in material and methods. (A) The lysine solution ( $50 \mu \mathrm{M}$, equivalence to $7.5 \mathrm{nmol}$ in $150 \mu \mathrm{l}$ test sample) were spent in serial double dilution. Each point represented the mean of 15 replicated. The limit of detection ( $\mathrm{LOD}=3.3 \times \mathrm{SDy} / \mathrm{slope})$ and limit of quantification $(\mathrm{LOQ}=10 \times \mathrm{SDy} / \mathrm{slope})$ of 0.22 and $0.66 \mathrm{nmol}$ were calculated for fluorescamine assay, respectively. (B) The Reduced Glutathione solution (100 $\mu \mathrm{M}$, equivalence to $17 \mathrm{nmol}$ in $170 \mu \mathrm{l}$ test sample) were spent in serial double dilution. Each point represented the mean of 12 replicated. The LOD and LOQ of 0.13 and $0.4 \mathrm{nmol}$ were calculated for Aldrithiol assay, respectively. (C) The Reduced Glutathione solution $(10 \mu \mathrm{M}$, equivalence to $1.7 \mathrm{nmol}$ in $170 \mu \mathrm{l}$ test sample) were spent in serial double dilution. Each point represented the mean of 3 replicated. 

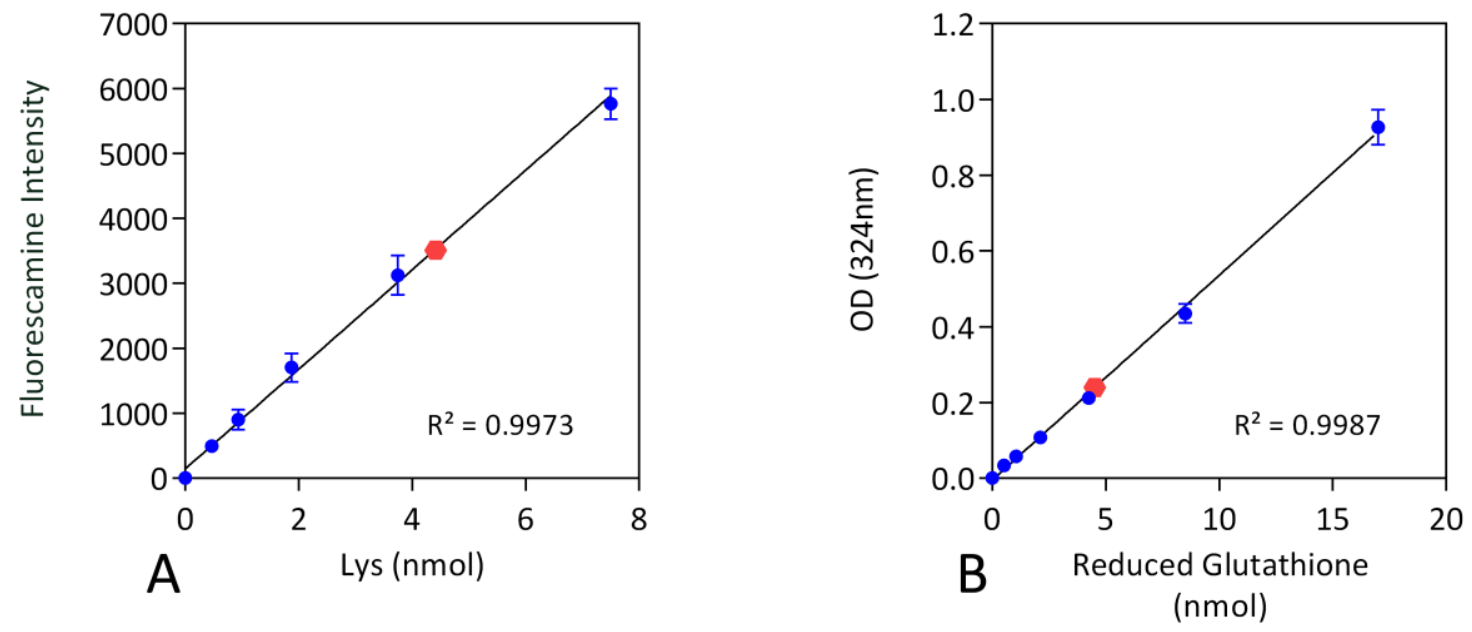

Figure S2. Quantification of Core Peptide. The calibration curves were prepared as described. The red points indicated the amount of core peptide with (A) Fluorescamine assay in $150 \mu 1$ (expected 4.5nmol) and (B) Aldrithiol assay in $170 \mu$ test volume (expected 5.1nmol). Each point represented the mean of 3 replicated. The resulted was calculated as below

- Amount determined in test volume for fluorescamine assay and Aldrithiol assay was 4.413 and $4.528 \mathrm{nmol}$ respectively.

- Amount determined in whole volume for fluorescamine assay and Aldrithiol assay was 7356 and $6659 \mathrm{nmol}$ respectively.

- $\quad$ The reduced content $=$ Amount. free sulfhydryl / Amount. primary amine $(6659 / 7356)=0.905$

- The discrepancy in the result shows that some amount of peptide was oxidize which could be happened during the purification or long term storage at refrigerator 

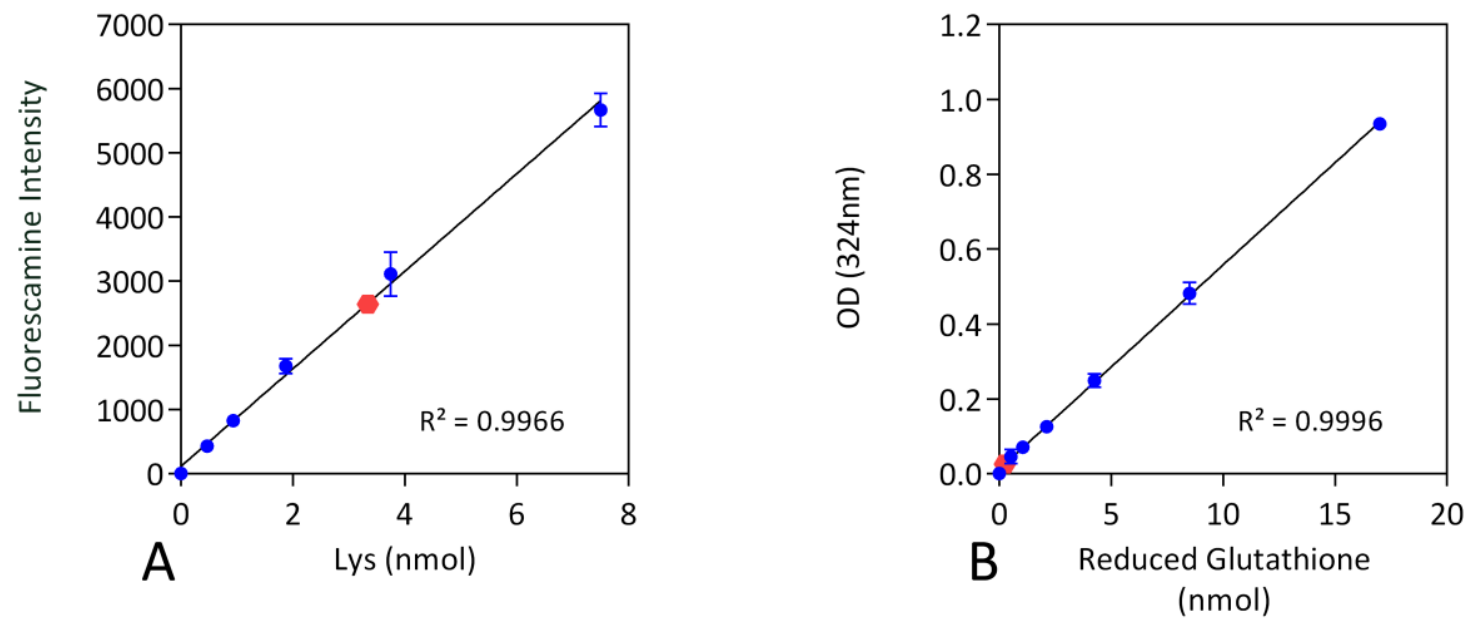

Figure S3. Quantification of purified PCP. The calibration curves were prepared as described. The red points indicated (A) the quantify primary amines of PCP and unreacted CP by Fluorescamine assay in $150 \mu$ volume and (B) indicated the free sulfhydryl of unreacted and remained peptide by Aldrithiol assay in $170 \mu \mathrm{l}$ test volume. Each point represented the mean of 3 replicated. The resulted was calculated as below

- Amount determined in test volume for fluorescamine assay and Aldrithiol assay was 3.341 and $0.252 \mathrm{nmol}$ respectively.

- Amount determined in whole volume for fluorescamine assay and Aldrithiol assay was 1113.6 and $74.3 \mathrm{nmol}$ respectively.

- Initial Amount of PEG was $24 \mathrm{mg}$ equivalence to $1200 \mathrm{nmol}$ (based on content of $86 \%$ activated PEG, according to manufacturer)

- $\quad$ Yield of reaction $=(($ Amount. PCP - Amount. CP $) /$ Amount. initial activated PEG $)$ $((1113.6-74.3) / 1200)=0.866$

- Efficiency of Peptide Purification

(Amount. initial CP - Amount. unreacted CP)/ Amount. initial CP $((6659-74.3) / 6659)=0.988$ 


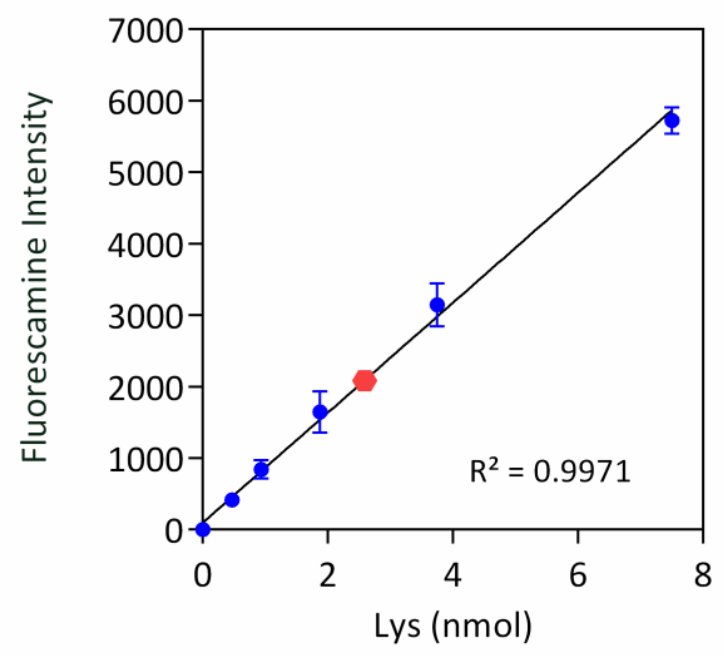

Figure S4. Quantification of PCP-SPDP Conjugation (Crude). The calibration curves were prepared as described. The red points indicated the quantify of primary amines which unreacted to SPDP by Fluorescamine assay in $150 \mu$ volume. Each point represented the mean of 3 replicated. The resulted was calculated as below

- Amount determined in test volume for fluorescamine assay was $2.589 \mathrm{nmol}$.

- Amount determined in whole volume (1 ml) for fluorescamine assay was $17.26 \mathrm{nmol}$.

- Amount of primary amines could be for PCP or Core peptide which remained from previous step (74.3 nmol).

- Yield of reaction $=(($ Amount. $\mathrm{PCP}+$ Amount. $\mathrm{CP})-$ Amount of primary amines $/$ (Amount.PCP + Amount. CP)

- $\quad((1113.6+74.3)-17.26) /(1113.6+74.3)=0.986$ 